\title{
Comparison and analysis of temperature rise characteristic of cable under the tunnel and pipe
}

\author{
Hong-Lei Li ${ }^{1}$, Zhen-Peng Zhang ${ }^{2}$, Shao-Xin Meng ${ }^{2}$, \\ Song-Hua Liu ${ }^{2}$ and Shu-Lian Xie ${ }^{3}$ \\ ${ }^{1}$ State Grid Shanghai Electric Power Research Institute, \\ Shanghai 200437, China; \\ ${ }^{2}$ Electric Power Research Institute, Wuhan 430074, China; \\ ${ }^{3}$ Wuhan Huazhong Numerical Control Co.,Ltd, Wuhan 430223, China; \\ E-mail:13971276891@163.com
}

\begin{abstract}
In order to analyze the difference between temperature rise and the current carrying capacity of cable lines in pipe and tunnel, this paper first analyzes the temperature rise under the laying condition of tunnel and pipe and the calculation method of load flow, and applied the same test current on a $220 \mathrm{kV}$ cable test circuit in pipe and in tunnel under laboratory conditions, by measuring the cable conductor temperature rise with thermocouple measurement technology. The applied current is the $24 \mathrm{~h}$ steady state current and the $24 \mathrm{~h}$ transient current, and the difference of the temperature rise of the cable is compared under the conditions of the tunnel and the pipe under the two current conditions. The temperature rise of the cable is compared with the two different ways of laying, and the influence of the steady state current and the time varying current on the temperature rise of the cable conductor is also compared.
\end{abstract}

Keywords: Power Cable; Temperature Rise; Pipe; Tunnel; Laying Condition.

\section{Introduction}

Long-distance characteristic of cable lines determines the diversity of their laying methods, which may include direct burying, cable trench, cable pipe, tunnel, bridge, underwater laying and so on. This will lead to different impact to the same cable under different environments. Due to the distribution characteristics and the diversity of laying environment along the cable lines, the temperature distribution along the cable lines is irregular.Because of different heat transfer conditions and environments, the temperature rise and current carrying capacity of the same cable lineare different under different laying methods.At the boundary of the two environments, due to the different heat dissipationconditions, the distribution temperature curve step and temperature rise curve step will mostly be formed. The laying environment and the location where the cable conductor temperature rise higherare often the bottleneck of the current carrying capacity of cable lines. 
Because of the good air flow and ventilation in the cable tunnel, the cable distribution temperature uniformity is higher and the cable heat dissipation is faster. So the current carrying capacity in the cable tunnel is higher. Cable pipe has a poor ventilation, poor cooling condition, and higher cableoperating temperature, it is often the bottleneck of entire cable line for current carrying capacity. The laying conditions have a decisive impact on cable conductor temperature rise, current carrying capacity calculation and load capacity for cable lines. Hence, it is necessary to analyze the difference of cable line temperature rise under different cable laying conditions to provide more accurate operational status information.

\section{TheoreticalAnalysis}

\subsection{Steady-state current cable temperature rise calculation}

The total heating value of the cable depends on the structure of the cable, including the geometrical parameters and material properties at each layer,load variables(including current, voltage and frequency, etc.), cable laying conditions and grounding loop current; The interior of the cable forms a thermal system taking cable surface as boundary, including the following three main heating sources under normal operation, as shown in Figure 2.1:

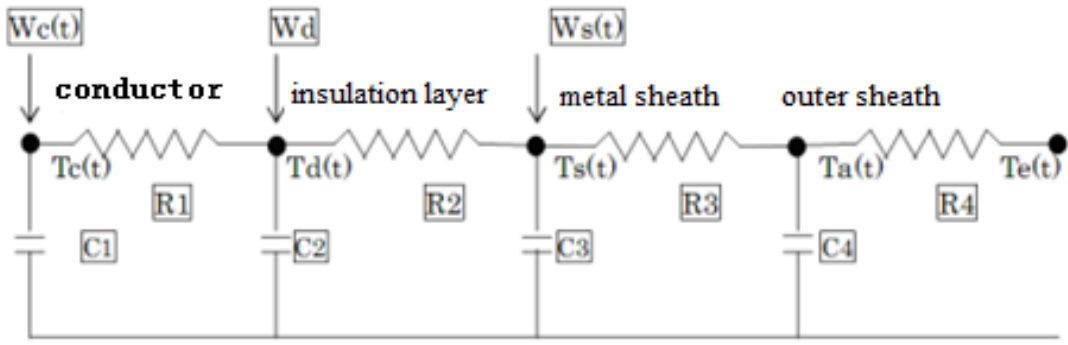

Fig.2.1.Cable structure layer temperature calculation model

(a)Conductor

$$
W c=I^{2} \cdot R_{0} \cdot\left[1+\alpha_{20}(T c c-20)\right] \cdot(1+Y s+Y p)
$$

Where $I$ is the current, $\mathrm{R}_{0}$ is the DC resistance of the conductor at $20^{\circ} \mathrm{C}, \alpha_{20}$ is the temperature coefficient of resistance of the conductor material, $T c c$ is the conductor temperature, $Y s$ is the skin effect factor; $Y P$ is the proximity effect factor. $R_{0}, \alpha_{20}$ and $Y s$ are determined by the cable structure material and power frequency; $Y p$ is related to the location of the cable laying.

(b)Insulating medium 


$$
W d=2 \pi \cdot f \cdot c \cdot U_{0}^{2} \cdot \operatorname{tg} \delta
$$

Where $f$ is the frequency of the power supply, $c$ is the capacitance of the cable, $U_{0}$ is the phase voltage, $\operatorname{tg} \delta$ is the insulation loss factor at the power supply system and operating temperature, which is distributed unevenly in the insulating layer and the distribution density is a function of the electric field strength, relatively higher near the conductor. For $220 \mathrm{kV}$ or more cables, the dielectric medium can reach the same level as the conductor loss. IEC distributes $50 \%$ of the dielectric medium loss at the inside and outside of insulating layer, thus the steady-state calculation is accurate. The dynamic calculation of conductor temperature must take into account the distribution of dielectric loss in the insulating layer.

(c) Metal sheath

$$
\mathrm{Ws}(\mathrm{t})=\lambda \cdot \mathrm{Wc}(\mathrm{t})
$$

The shielding loss is related to the phase arrangement of the circuit itself and the peripheral circuit. This loss is small in the case of shielded single-ended grounding and well-balanced cross-bondingconnection grounding; In the opposite case, a significant shielding loop may be caused. Its loss may be large.

$$
\begin{gathered}
T_{c}(t+\Delta t)=\frac{\Delta t}{C_{1}}\left[W_{c}(t)-\frac{T_{c}(t)-T_{d}(t)}{R_{1}}\right]+T_{c}(t) \\
T_{d}(t+\Delta t)=\frac{\Delta t}{C_{2}}\left[W_{d}(t)-\frac{T_{c}(t)-T_{d}(t)}{R_{1}}-\frac{T_{d}(t)-T_{s}(t)}{R_{2}}\right]+T_{d}(t) \\
T s(t+\Delta t)=\frac{\Delta t}{C_{3}}\left[W_{s}(t)+\frac{T_{d}(t)-T_{s}(t)}{R_{2}}-\frac{T_{s}(t)-T_{a}(t)}{R_{3}}\right]+T_{s}(t) \\
T_{a}(t+\Delta t)=\frac{\Delta t}{C_{4}}\left[\frac{T_{s}(t)-T_{a}(t)}{R_{3}}-\frac{T_{a}(t)-T_{e}(t)}{R_{4}}\right]+T_{a}(t)
\end{gathered}
$$

In the formulas:

$T_{c}, T_{d}, T_{s}, T_{a}$ and $T_{a}$ are conductor temperature, insulation layer temperature, metal sheath temperature, outer sheath temperature and real-time measurement temperature of the cable outer sheath. Unit: ${ }^{\circ} \mathrm{C}$.

$C_{1}, C_{2}, C_{3}$ and $C_{4}$ are the thermal capacity of the conductor, thermal capacity of the insulation layer, the thermal capacity of the metal sheath and the thermal capacity of the outer sheath. Unit: $\mathrm{J} / \mathrm{K} \cdot \mathrm{m}$.

$W_{C}, W_{d}$ and $W_{s}$ are the thermal loss of the conductor, the thermal loss of the insulation layer, and the thermal loss of the outer sheath. Unit: W/ m. 
$R_{1}, R_{2}, R_{3}$ and $R_{4}$ are the thermal resistance of the conductor, the thermal resistance of the insulation layer, the thermal resistance of the metal sheath and the thermal resistance of the outer sheath. Unit: $\mathrm{K} \bullet \mathrm{m} / \mathrm{W}$.

\section{Test}

\subsection{Test arrangement}

The experimental study was carried out on XLPE insulated power cables with a rated voltage of $220 \mathrm{kV}$ and section of $2500 \mathrm{~mm}^{2}$ and length of $15 \mathrm{~m}$. Conductor thermocouple and outer sheath thermocouple are used to measure the conductor and outer sheath temperature of the cable directly. The size of the conductor thermocouple and its installation method shall follow IEC standard. Install an outer sheath thermocouple on the outer sheath at a location less than $0.2 \mathrm{~m}$ from the conductor thermocouple installation location.

The cables are laid in tunnel and pipe environment separately, where the cables are laid horizontally in the tunnel at a height of 50 mmabove the ground and the total length of the simulated tunnel is $18 \mathrm{~m}$. In cable pipeconditions, the pipelength is $12 \mathrm{~m}$ and the cable is buried in $0.8 \mathrm{~m}$ deep soil after laying.

\subsection{Test process and Data}

\subsubsection{Steady-state load current test}

$220 \mathrm{kV}$ cable samples were placed intunnel and cable pipe conditions and were applied with steady load current $2500 \mathrm{~A}$ for $24 \mathrm{~h}$; Record the cable conductor temperature and outer sheath temperature. As shown in Table 3.1 and Fig.3.1.

(a)Tunnel laying

Cable starting temperature is $30.9^{\circ} \mathrm{C}$. Apply steady-state load current 2500 A for $24 \mathrm{~h}$. Use cable-specific temperature recorder to record inner and outer sheath thermocouple measured values and cable conductor thermocouple measured values for $24 \mathrm{~h}$. The measured conductor temperature of cable is $64.3^{\circ} \mathrm{C}$ at $24 \mathrm{~h}$, corresponding to $34.2^{\circ} \mathrm{C}$, the conductor temperature of the outer sheath thermocouple measured temperature.

(b) Cable pipe laying

Cable starting temperature is $33.9^{\circ} \mathrm{C}$. Apply steady-state load current 2500 A for $24 \mathrm{~h}$. Use cable-specific temperature recorder to record inner and outer sheath thermocouple measured values and cable conductor thermocouple measured values for $24 \mathrm{~h}$. The cable measured conductor temperature is $77.4^{\circ} \mathrm{C}$ at $24 \mathrm{~h}$, corresponding to $52.8^{\circ} \mathrm{C}$, the conductor temperature of the outer sheath thermocouple measured temperature. 
Table 3.1. Test data under steady-state current condition

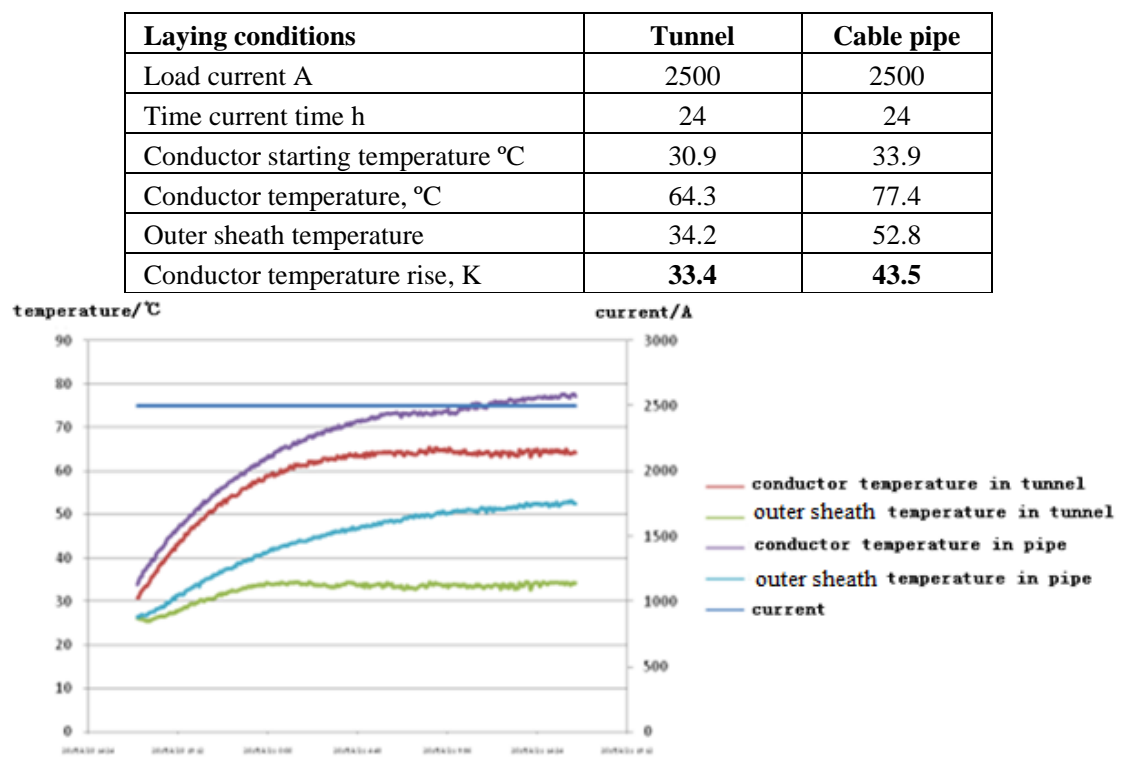

Fig.3.1. Curve of steady-state current test data

\subsubsection{Time-varying load current test}

$220 \mathrm{kV}$ cable samples were placed in tunnel and pipeconditions. Apply $24 \mathrm{~h}$ variable load current, with the maximum current value of 3200A. The current changes every $2 \mathrm{~h}$. Record the cable conductor temperature and outer sheath temperature.

\section{(a)Tunnel laying}

As shown in Table 2 and in Fig.3.6. Cable starting temperature is $24.2^{\circ} \mathrm{C}$. Apply load varying current for $24 \mathrm{~h}$. Use cable-specific temperature recorder to record inner and outer sheath thermocouple measured values and cable conductor thermocouple measured values for $24 \mathrm{~h}$. The measured max. conductor temperature of cable is $72.0^{\circ} \mathrm{C}$ at $24 \mathrm{~h}$, corresponding to $36.5^{\circ} \mathrm{C}$, the conductor temperature of the outer sheath thermocouple measured temperature.

(b) Cable pipe laying

Cable starting temperature is $32.2^{\circ} \mathrm{C}$. Apply load-varying current for $24 \mathrm{~h}$. Use cable-specific temperature recorder to record inner and outer sheath thermocouple measured values and cable conductor thermocouple measured values for $24 \mathrm{~h}$. The measured max. conductor temperature of cable is $78.1^{\circ} \mathrm{C}$ at $24 \mathrm{~h}$, corresponding to $48.7^{\circ} \mathrm{C}$, the conductor temperature of the outer sheath thermocouple measured temperature. 
Table 3.2.Test data under the conditions of time-varying current

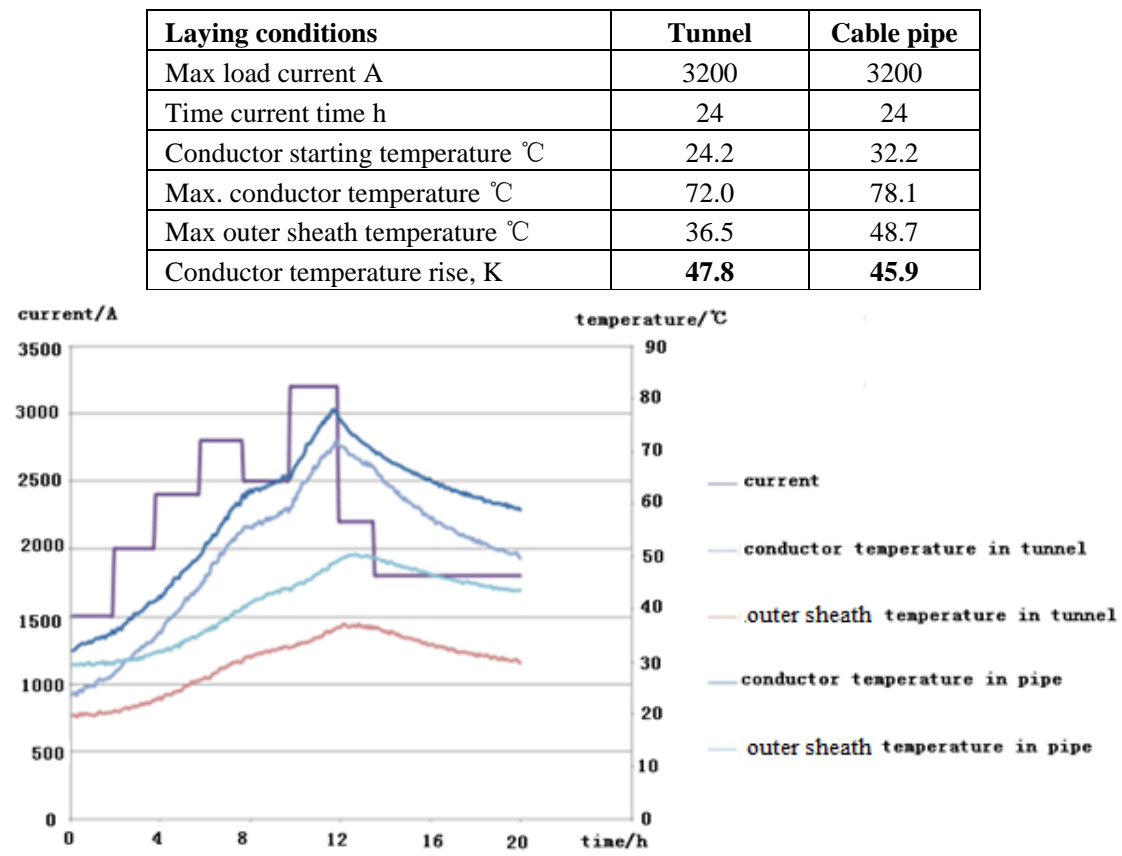

Fig.3.2. Curve of time-varying current test data

\section{Test Data Analysis and Conclusions}

By analyzing the experimental data, when the steady-state load current is applied to the cable line for $24 \mathrm{~h}$, the temperature of the conductor tends to be stable after applying steady-state current for more than $12 \mathrm{~h}$. The difference between the highest conductor temperature under the tunnel condition and the highest conductor temperature under cable pipe is $-13.1 \mathrm{~K}$, corresponding to outer sheath temperature difference $-18.6 \mathrm{~K}$.

When the load-varying current is applied to the cable line for $24 \mathrm{~h}$, the followability of cable conductor temperature changewith the current change under tunnel condition and cable pipe condition is good. The difference between the highest conductor temperature and the highest conductor temperature under tunnel conditions is $-6.1 \mathrm{~K}$, which corresponds to outer sheath temperature difference of $-12.2 \mathrm{~K}$.The temperature rise of the cable conductor under tunnel condition is $33.4 \mathrm{~K}$ after applying $24 \mathrm{~h}$ steady-state load current; The temperature rise of the cable conductor is $43.3 \mathrm{~K}$ under cable pipe condition, where the temperature difference is $-9.9 \mathrm{~K}$.

The cable conductor temperature rise under tunnel condition is $47.8 \mathrm{~K}$ after applying the load-varying current for $24 \mathrm{~h}$, and the temperature rise of the cable 
conductor under cable pipe condition is $45.9 \mathrm{~K}$, where the temperature rise differenceis $1.9 \mathrm{~K}$.

This paper studied the conductor temperature variation of $220 \mathrm{kV}$ cable under the condition of tunnel and cable pipe and steady-state load current and time-varying current. The results show that for steady-state current, the cable line under tunnel condition has a good heat dissipation and the outer sheath temperature variation caused by the conductor temperature rise is small. Thus, its current carrying capacity is high.For cable line laid in pipe, the heat dissipation is poor.The outer sheath temperature rise caused by the conductor temperature rise is big,therefore the current carrying capacity is low. But for time-varying current, the difference of current carrying capacity because ofcurrent changes between tunnel laying andpipe laying is smaller.

\section{References}

1. Optimization of power transmission capability of underground cable systems using thermal monitoring[R]. Report of CIGRE WG B1-02: 2004.

2. Construction, laying and installation techniques for extruded and selfcontained fluid filled cable systems[R]. Report of CIGRE WG 21-17: 2001.

3. D. A. Douglass and A. A. Edris. Real-time monitoring and dynamic thermal rating of power transmission circuits[J]. IEEE Trans. Power Del. vol. 11, no.3, pp. 1407-1417, Jul. 1996.

4. IEC62067. Power cable with extruded insulation and their accessories for rated voltages above $150 \mathrm{kV}(\mathrm{Um}=170 \mathrm{kV})$ up to $500 \mathrm{kV}(\mathrm{Um}=550 \mathrm{kV})$ Test methods and requirements[S], 2001.

5. ZHANG Zhenpeng, ZHAO Jiankang, RAO Wenbin, et al. Validate Test for the Calculation congruity of Distributed temperature Sensing System[J], High Voltage Engineering, 2012, 38(6):1362-1367. 